\title{
The Electrostatic Interaction Between Two Charged Surfaces with Mixed Boundary Conditions
}

The electrostatic interaction between two parallel, planar charged surfaces with periodic mixed boundary conditions is analyzed. The Poisson - B oltzmann equation (PBE ) governing the potential distribution of the system under consideration is solved by the Fourier transformation method coupled with the convolution theory. The present approach provides an efficient way to solve a PBE with a general mixed boundary condition. On the basis of this approach, an analytical expression for the electrostatic interaction energy is derived. In addition to its generality, the method proposed is very convenient for the description of the shift of the relative position of two surfaces. $\odot 1996$ Academic Press, Inc.

Key Words: rigid surfaces, parallel plates; Poisson-Boltzmann equation, linearized; boundary conditions, general, mixed, periodic; interaction energy, electrostatic; integral transform, Fourier, convolution.

\section{INTRODUCTION}

The magnitude of the electrostatic interaction between two charged surfaces depends on the electrical potential distribution governed by the Poisson-Boltzmann equation (PBE). Often, a charged surface is assumed to remain at either constant potential or constant charge density. This assumption can be unrealistic for several classes of surfaces in practice. Typical example includes biocolloids, in which the surface charges are due to the dissociation of the functional groups they bear. The distribution of these functional groups can be position dependent, and their degree of dissociation depends largely on the condition of the environment. In this case, the boundary condition associated with the corresponding PBE is nonlinear, and the surface charge density is a nonlinear function of potential (1). A widely adopted approach for this problem is the Newton Raphson method, in which a nonlinear boundary condition is first approximated by a linear expression, followed by an iterative procedure (2). Here, the problem under consideration is equivalent to a mixed boundary problem in which a linear combination of the potential and its derivative is specified on the boundary. In the present study a systematic approach based on the Fourier transform, coupled with the convolution theory (3), for the resolution of a PBE subject to a general mixed boundary condition is suggested. We show that this technique provides an efficient way of portraying periodic mixed boundary conditions and is readily applicable to the estimation of the interaction energy of two charged surfaces.

\section{ANALYSIS}

Let us consider two parallel, planar charged surfaces immersed in an electrolyte solution. Under the Debye-Hückel condition, the electrostatic potential is governed by the Poisson-Boltzmann equation $(4,5)$

$$
\frac{\partial^{2} \Psi}{\partial x^{2}}+\frac{\partial^{2} \Psi}{\partial y^{2}}+\frac{\partial^{2} \Psi}{\partial z^{2}}=\Psi
$$

where $\Psi=e \phi / k T, x=\kappa \tilde{x}, y=\kappa \tilde{y}, z=\kappa \tilde{z}$, and $\kappa^{2}=\left(e^{2} \sum_{i} z_{i}^{2} n_{i 0}\right) /$ $\left(\epsilon_{\mathrm{r}} \epsilon_{0} k T\right)$. In these expressions, $(\tilde{x}, \tilde{y}, \tilde{z})$ denotes the position of a point in space, $\phi$ is the electrostatic potential, $k$ and $e$ are, respectively, the Boltzmann constant and the elementary charge, $T$ is the absolute temperature, $z_{i}$ and $n_{i 0}$ are, respectively, the valence and the concentration of the $i$ th ion species in the bulk liquid phase, and $\epsilon_{\mathrm{r}}$ and $\epsilon_{0}$ are the relative permittivity of solution and the permittivity of the vacuum, respectively. Here, $\Psi$ denotes the dimensionless electrostatic potential, and $1 / \kappa$ is the Debye length. For convenience, one of the two surfaces is placed at $z=-L$ and the other at $z=L$. We consider the general boundary conditions

$$
\begin{aligned}
& f_{1}(x, y) \Psi(x, y, z)+g_{1}(x, y) \frac{\partial \Psi(x, y, z)}{\partial z}=h_{1}(x, y), \quad z=-L \\
& f_{2}(x, y) \Psi(x, y, z)+g_{2}(x, y) \frac{\partial \Psi(x, y, z)}{\partial z}=h_{2}(x, y), \quad z=L .
\end{aligned}
$$

Suppose that all the functions in [2] are periodic with periods $2 L_{x}$ and $2 L_{y}$ in the $x$ and $y$ directions, respectively. The general solution to [1] can be expressed as

$$
\begin{aligned}
\Psi(x, y, z)=\sum_{p} \sum_{q}\left[\alpha_{p q} \exp (\right. & \left.-I_{p q}(z+L)\right) \\
& \left.+\beta_{p q} \exp \left(I_{p q}(z-L)\right)\right] \psi(p, x) \psi(q, y),
\end{aligned}
$$

where $I_{p q}=\sqrt{1+\left(\pi p / L_{x}\right)^{2}+\left(\pi q / L_{y}\right)^{2}}, \psi(p, x)=\exp \left(j \pi p x / L_{x}\right), \psi(q$, $y)=\exp \left(j \pi q y / L_{y}\right)$, and $j=\sqrt{-1}$. Unless it is specified, the indices $p$ and $q$ range from $-\infty$ to $\infty$. Expressing $\Psi$ and $\partial \Psi / \partial z$ at $z=-L$ in Fourier series representation gives

$$
\begin{gathered}
\Psi(x, y,-L)=\sum_{p} \sum_{q} \hat{\Psi}_{-L, p, q} \psi(p, x) \psi(q, y) \\
\left(\frac{\partial \Psi}{\partial z}\right)_{z=-L}=\sum_{p} \sum_{q}\left(\frac{\partial \hat{\Psi}}{\partial z}\right)_{-L, p, q} \psi(p, x) \psi(q, y) .
\end{gathered}
$$

We define

$$
\left[\begin{array}{c}
\hat{\Psi}_{-L, p, q} \\
(\partial \hat{\Psi} / \partial z)_{-L, p, q}
\end{array}\right]=\overline{\bar{P}}_{-L, p, q}\left[\begin{array}{c}
\alpha_{p q} \\
\beta_{p q}
\end{array}\right],
$$

where

$$
\bar{P}_{-L, p, q}=\left[\begin{array}{cc}
1 & \exp \left(-2 I_{p q} L\right) \\
-I_{p q} & I_{p q} \exp \left(-2 I_{p q} L\right)
\end{array}\right] .
$$


Here, a symbol with two overhead bars denotes a matrix. Taking the Fourier transform on [2] for $z=-L$ and applying the convolution theorem (3), we have

$$
\sum_{p} \sum_{q}\left(\hat{f}_{1, m-p, n-q} \hat{\Psi}_{-L, p, q}+\hat{g}_{1, m-p, n-q}\left(\frac{\partial \hat{\Psi}}{\partial z}\right)_{-L, p, q}\right)=4 L_{x} L_{y} \hat{h}_{1, m, n},
$$

where $\hat{f}_{1, p, q}$ and $\hat{g}_{1, p, q}$ are the Fourier transforms of $f_{1}$ and $g_{1}$, respectively. Similarly, we have the following expressions at $z=L$ :

$$
\begin{gathered}
\sum_{p} \sum_{q}\left(\hat{f}_{2, m-p, n-q} \hat{\Psi}_{L, p, q}+\hat{g}_{2, m-p, n-q}\left(\frac{\partial \hat{\Psi}}{\partial z}\right)_{L, p, q}\right)=4 L_{x} L_{y} \hat{h}_{2, m, n} \\
{\left[\begin{array}{c}
\hat{\Psi}_{L, p, q} \\
(\partial \hat{\Psi} / \partial z)_{L, p, q}
\end{array}\right]=\overline{\bar{P}}_{L, p q}\left[\begin{array}{c}
\alpha_{p q} \\
\beta_{p q}
\end{array}\right],} \\
\overline{\bar{P}}_{L, p q}=\left[\begin{array}{cc}
\exp \left(-2 I_{p q} L\right) & 1 \\
-I_{p q} \exp \left(-2 I_{p q} L\right) & I_{p q}
\end{array}\right] .
\end{gathered}
$$

The coefficients $\alpha_{p q}$ and $\beta_{p q}$ can be obtained by solving [8] and [9] simultaneously. If the rate of convergence of the right-hand side of [3] is sufficiently fast, it can be approximated by

$$
\begin{aligned}
\Psi \simeq \sum_{p=-M}^{M} \sum_{q=-N}^{N}\left[\alpha_{p q} \exp (-\right. & \left.I_{p q}(z+L)\right) \\
& \left.+\beta_{p q} \exp \left(I_{p q}(z-L)\right)\right] \psi(p, x) \psi(q, y) .
\end{aligned}
$$

In this case [8] and [9] can be written as

$$
\left[\begin{array}{c}
\overline{\bar{M}}_{1} \bar{v}_{-L} \\
\overline{\bar{M}}_{2} \bar{v}_{L}
\end{array}\right] \simeq\left[\begin{array}{l}
\bar{N}_{1} \\
\bar{N}_{2}
\end{array}\right]
$$

where

$$
\bar{v}_{L}=\left[\bar{v}_{L,-M}, \bar{v}_{L,-M+1}, \ldots, \bar{v}_{L, M}\right]^{\mathrm{T}},
$$

$$
\begin{aligned}
\bar{v}_{L, k}=\left[\hat{\Psi}_{L, k,-N},\left(\frac{\partial \hat{\Psi}}{\partial z}\right)_{L, k,-N},\right. & \\
& \left.\hat{\Psi}_{L, k,-N+1},\left(\frac{\partial \hat{\Psi}}{\partial z}\right)_{L, k,-N+1}, \ldots, \hat{\Psi}_{L, k, N},\left(\frac{\partial \hat{\Psi}}{\partial z}\right)_{L, k, N}\right]^{\mathrm{T}}
\end{aligned}
$$

$$
\begin{gathered}
\bar{N}_{1}=4 L_{x} L_{y}\left[\bar{h}_{1,-M_{p}}, \overline{\hat{h}}_{1,-M_{p}+1}, \ldots, \overline{\hat{h}}_{1, M_{p}}\right]^{\mathrm{T}}, \\
\overline{\hat{h}}_{1, k}=\left[\hat{h}_{1, k,-N_{p}}, \hat{h}_{1, k,-N_{p}+1}, \ldots, \hat{h}_{1, k, N_{p}}\right]^{\mathrm{T}}, \\
\bar{N}_{2}=4 L_{x} L_{y}\left[\overline{\hat{h}}_{2,-M_{p}}, \overline{\hat{h}}_{2,-M_{p}+1}, \ldots, \overline{\hat{h}}_{2, M_{p}}\right]^{\mathrm{T}}, \\
\overline{\hat{h}}_{2, k}=\left[\hat{h}_{2, k,-N_{p}}, \hat{h}_{2, k,-N_{p}+1}, \ldots, \hat{h}_{2, k, N_{p}}\right]^{\mathrm{T}}, \\
M_{p} \geqslant M, \quad N_{p} \geqslant N,
\end{gathered}
$$

where the superscript $\mathrm{T}$ denotes the transpose. According to [6] and [10], $\bar{v}_{-L}$ and $\bar{v}_{L}$ and $\alpha_{p q}$ and $\beta_{p q}$ are related by

$$
\bar{v}_{-L}=\bar{P}_{-L} \bar{v}_{\alpha \beta} \quad \text { and } \quad \bar{v}_{L}=\overline{\bar{P}}_{L} \bar{v}_{\alpha \beta}
$$

where

$$
\begin{aligned}
\bar{v}_{\alpha \beta} & =\left[\bar{v}_{\alpha \beta,-M}, \bar{v}_{\alpha \beta,-M+1}, \ldots, \bar{v}_{\alpha \beta, M}\right]^{\mathrm{T}}, \\
\bar{v}_{\alpha \beta, k} & =\left[\alpha_{k,-N}, \beta_{k,-N}, \alpha_{k,-N+1}, \beta_{k,-N+1}, \ldots, \alpha_{k, N}, \beta_{k, N}\right]^{\mathrm{T}},
\end{aligned}
$$

and $\overline{\bar{P}}_{L(-L)}$ is the block-diagonal matrix

$$
\overline{\bar{P}}_{L}=\operatorname{diag}\left[\overline{\bar{P}}_{L,-M,-N}, \overline{\bar{P}}_{L,-M,-N+1}, \ldots, \overline{\bar{P}}_{L, M, N}\right] .
$$

Equation [13] can be rewritten as

$$
\left[\begin{array}{c}
\overline{\bar{M}}_{1} \overline{\bar{P}}_{-L} \\
\overline{\bar{M}}_{2} \overline{\bar{P}}_{L}
\end{array}\right] \bar{v}_{\alpha \beta} \simeq\left[\begin{array}{l}
\bar{N}_{1} \\
\bar{N}_{2}
\end{array}\right]
$$

We define the optimal solution to this equation, $\bar{v}_{\alpha, \beta}^{*}$, as the one at which the expression

$$
C\left(\bar{v}_{\alpha, \beta}\right)=\left\|\left[\begin{array}{c}
\overline{\bar{M}}_{1} \overline{\bar{P}}_{-L} \\
\overline{\bar{M}}_{2} \bar{P}_{L}
\end{array}\right] \bar{v}_{\alpha \beta}-\left[\begin{array}{c}
\bar{N}_{1} \\
\bar{N}_{2}
\end{array}\right]\right\|^{2}
$$

is minimized, where $\left\|\left[u_{1}, u_{2}, u_{3}, \ldots, u_{n}\right]\right\|^{2}=\sum_{i=1}^{n} u_{i}^{2}$. It can be shown that

$$
\begin{aligned}
\bar{v}_{\alpha \beta}^{*}=\left(\overline{\bar{P}}_{-L}^{\mathrm{T}} * \overline{\bar{M}}_{1}^{\mathrm{T}} *\right. & \overline{\bar{M}}_{1} \overline{\bar{P}}_{-L} \\
& \left.+\bar{P}_{L}^{\mathrm{T} *} \overline{\bar{M}}_{2}^{\mathrm{T} *} \overline{\bar{M}}_{2} \bar{P}_{L}\right)^{-1}\left(\bar{P}_{-L}^{\mathrm{T}} * \bar{M}_{1}^{\mathrm{T}} * N_{1}+\bar{P}_{L}^{\mathrm{T} *} \overline{\bar{M}}_{2}^{\mathrm{T} *} \bar{N}_{2}\right),
\end{aligned}
$$

where the superscript $T^{*}$ denotes the conjugate transpose.

\subsection{Electrostatic Free Energy}

We consider the general expression for the electrostatic free energy $E$

$$
E(l)=\int_{\Omega_{-L}} S_{-L}(x, y) \Psi \frac{\partial \Psi}{\partial z} d \Omega_{-L}+\int_{\Omega_{L}} S_{L}(x, y) \Psi \frac{\partial \Psi}{\partial z} d \Omega_{L}
$$

where $l(=2 L)$ is the distance between two surfaces, $\Omega_{-L}$ and $\Omega_{L}$ are the regions defined by one period on $z=-L$ and $z=L$, respectively, and $S_{-L}(x$, $y$ ) and $S_{L}(x, y)$ are the weighting factors which are functions of boundary conditions. For example, if the surface at $z=-L$ is at constant potential, $S_{-L}(x, y)=1 / 2$, and if it is at constant charge density, $S_{-L}(x, y)=-1 / 2$ (6). If some portion of the surface at $z=-L$ is at constant potential and the rest of the surface at constant charge density, then $S_{-L}(x, y)=1 / 2$ for the former and $S_{-L}=-1 / 2$ for the latter. It can be shown that

$$
\begin{aligned}
E= & \frac{1}{16 L_{x}^{2} L_{y}^{2}} \sum_{m} \sum_{n} \hat{S}_{-L, m, n}^{\mathrm{T}} \sum_{i} \sum_{j} \hat{\Psi}_{-L, m-i, n-j}\left(\frac{\partial \Psi}{\partial z}\right)_{-L, i, j} \\
& +\frac{1}{16 L_{x}^{2} L_{y}^{2}} \sum_{m} \sum_{n} \hat{S}_{L, m, n}^{\mathrm{T}} \sum_{i} \sum_{j} \hat{\Psi}_{L, m-i, n-j}\left(\frac{\partial \Psi}{\partial z}\right)_{L, i, j} \\
= & \frac{1}{16 L_{x}^{2} L_{y}^{2}} \overline{\hat{S}}_{-L}^{\mathrm{T}} \cdot\left[\left(\overline{\bar{C}}_{1} \overline{\bar{P}}_{-L} \bar{v}_{\alpha \beta}^{*}\right) \star\left(\overline{\bar{C}}_{2} \overline{\bar{P}}_{-L} \bar{v}_{\alpha \beta}^{*}\right)\right] \\
& +\frac{1}{16 L_{x}^{2} L_{y}^{2}} \bar{S}_{L}^{\mathrm{T}} * \cdot\left[\left(\overline{\bar{C}}_{1} \bar{P}_{L} \bar{v}_{\alpha \beta}^{*}\right) \star\left(\overline{\bar{C}}_{2} \bar{P}_{L} \bar{v}_{\alpha \beta}^{*}\right)\right],
\end{aligned}
$$


where $\star$ denotes the convolution operator, - represents the inner product, $\hat{S}_{-L}$ and $\hat{S}_{L}$ are, respectively, the Fourier coefficients of $S_{-L}$ and $S_{L}$, and

$$
\begin{aligned}
& \overline{\bar{C}}_{1}=\left[\begin{array}{ccccccc}
1 & 0 & 0 & 0 & 0 & 0 & \cdots \\
0 & 0 & 1 & 0 & 0 & 0 & \cdots \\
0 & 0 & 0 & 0 & 1 & 0 & \cdots \\
& & & \vdots & & &
\end{array}\right], \\
& \overline{\bar{C}}_{2}=\left[\begin{array}{ccccccc}
0 & 1 & 0 & 0 & 0 & 0 & \cdots \\
0 & 0 & 0 & 1 & 0 & 0 & \cdots \\
0 & 0 & 0 & 0 & 0 & 1 & \cdots \\
& & & \vdots & & &
\end{array}\right] .
\end{aligned}
$$

The matrices $\overline{\bar{C}}_{1}$ and $\overline{\bar{C}}_{2}$ are used to recover $\overline{\hat{\Psi}}_{-L}$ and $(\partial \overline{\hat{\Psi}} / \partial z)_{-L}$ from $\bar{v}_{-L}$, and $\bar{\Psi}_{L}$ and $(\partial \overline{\hat{\Psi}} / \partial z)_{L}$ from $\bar{v}_{L}$, respectively. On the basis of [20] and [22], and the relation

$$
\bar{P}_{L, p q}=\left[\begin{array}{cc}
1 & 0 \\
0 & -1
\end{array}\right] \bar{P}_{-L, p q}\left[\begin{array}{ll}
0 & 1 \\
1 & 0
\end{array}\right]
$$

it can be shown that $\bar{P}_{-L}$ is the only term in [22] which is $L$-dependent. For a constant potential/charge density problem, $S_{-L}(x, y)$ and $S_{L}(x, y)$ are constant, the convolution in [22] can be replaced by an inner product, and [22] reduces to

$$
E=\frac{S_{-L}}{4 L_{x} L_{y}}\left[\left(\overline{\bar{C}}_{1} \bar{P}_{-L} \bar{v}_{\alpha \beta}^{*}\right) \cdot\left(\overline{\bar{C}}_{2} \bar{P}_{-L} \bar{v}_{\alpha \beta}^{*}\right)\right]+\frac{S_{L}}{4 L_{x} L_{y}}\left[\left(\overline{\bar{C}}_{1} \bar{P}_{L} \bar{v}_{\alpha \beta}^{*}\right) \cdot\left(\overline{\bar{C}}_{2} \overline{\bar{P}}_{L} \bar{v}_{\alpha \beta}^{*}\right)\right]
$$

\subsection{Position Shift}

Suppose that the plane at $z=L$ is shifted $d_{x}$ units in the $x$ direction and $d_{y}$ units in the $y$ direction for a fixed distance between two surfaces. In this case the boundary condition expressed by [2] becomes

$$
\begin{gathered}
f_{1}(x, y) \Psi(x, y, z)+g_{1}(x, y) \frac{\partial \Psi(x, y, z)}{\partial_{\bar{z}}}=h_{1}(x, y), \quad z=-L \\
f_{2}\left(x+d_{x}, y+d_{y}\right) \Psi(x, y, z)+g_{2}\left(x+d_{x}, y+d_{y}\right) \frac{\partial \Psi(x, y, z)}{\partial_{\bar{z}}} \\
=h_{2}\left(x+d_{x}, y+d_{y}\right), \quad z=L, \quad
\end{gathered}
$$

we have

$$
\sum_{p=-M}^{M} \sum_{q=-N}^{N}\left(\hat{f}_{1, m-p, n-q} \hat{\Psi}_{-L, p, q}+\hat{g}_{1, m-p, n-q}\left(\frac{\partial \hat{\Psi}}{\partial z}\right)_{-L, p, q}\right)=4 L_{x} L_{y} \hat{h}_{1, m, n}
$$

and

$$
\begin{gathered}
\sum_{p=-M}^{M} \sum_{q=-N}^{N} D_{m-p, n-q}\left(\hat{f}_{2, m-p, n-q} \hat{\Psi}_{L, p, q}+\hat{g}_{2, m-p, n-q}\left(\frac{\partial \hat{\Psi}}{\partial z}\right)_{L, p, q}\right) \\
=D_{m, n} 4 L_{x} L_{y} \hat{h}_{2, m, n}, \\
m=-M_{p},-M_{p}+1,-M_{p}+2, \ldots, M_{p}, \quad M_{p} \geqslant M, \\
n=-N_{p},-N_{p}+1,-N_{p}+2, \ldots, N_{p}, \quad N_{p} \geqslant N,
\end{gathered}
$$

where $D_{p, q}=\exp \left(j p \pi d_{x} / L_{x}\right) \exp \left(j q \pi d_{y} / L_{y}\right)$. The shift of the surface at $z$ $=L$ is characterized by the factor $D_{p, q}$. Equation [13] becomes

$$
\left[\begin{array}{c}
\overline{\bar{M}}_{1} v_{-L} \\
\overline{\bar{D}}_{p} \overline{\bar{M}}_{2} \overline{\bar{D}} v_{L}
\end{array}\right] \simeq\left[\begin{array}{c}
\bar{N}_{1} \\
\overline{\bar{D}}_{p} \bar{N}_{2}
\end{array}\right]
$$

or

$$
\left[\begin{array}{c}
\overline{\bar{M}}_{1} v_{-L} \\
\overline{\bar{M}}_{2} \overline{\bar{D}}_{L}
\end{array}\right]=\left[\begin{array}{c}
\overline{\bar{M}}_{1} \overline{\bar{P}}_{-L} \\
\bar{M}_{2} \overline{\bar{D}} \bar{P}_{L}
\end{array}\right] \bar{v}_{\alpha \beta} \simeq\left[\begin{array}{c}
\bar{N}_{1} \\
\bar{N}_{2}
\end{array}\right]
$$

In these expressions $\overline{\bar{D}}$ is the block-diagonal matrix with diagonal elements $\overline{\bar{D}}_{s}, s=M,(M-1),(M-2), \ldots,-(M-1),-M$, with

$$
\overline{\bar{D}}_{s}=\operatorname{diag}\left[D_{s, N_{p}} \overline{\bar{I}}_{2 \times 2}, D_{s, N_{p}-1} \overline{\bar{I}}_{2 \times 2}, D_{s, N_{p}-2} \overline{\bar{I}}_{2 \times 2}, \ldots, D_{s,-N_{p}} \overline{\bar{I}}_{2 \times 2}\right]
$$

$\overline{\bar{I}}_{2 \times 2}$ is the $2 \times 2$ identity matrix and $\overline{\bar{D}}_{p}$ is the diagonal matrix with diagonal elements $\overline{\bar{D}}_{k}, k=-M_{p},-\left(M_{p}-1\right),-\left(M_{p}-2\right), \ldots,\left(M_{p}-1\right), M_{p}$, with

$$
\overline{\bar{D}}_{k}=\operatorname{diag}\left[D_{k,-N_{p}}, D_{k,-N_{p}+1}, D_{k,-N_{p}+2}, \ldots, D_{k, N_{p}}\right]
$$

The optimal solution to [29] is

$$
\begin{aligned}
\bar{v}_{\alpha \beta}^{*}=\left(\overline{\bar{P}}_{-L}^{\mathrm{T} *} \overline{\bar{M}}_{1}^{\mathrm{T} *} \overline{\bar{M}}_{1} \overline{\bar{P}}_{-L}+\overline{\bar{P}}_{L}^{\mathrm{T} *} \overline{\bar{D}}^{\mathrm{T} *} \overline{\bar{M}}_{2}^{\mathrm{T} *} \overline{\bar{M}}_{2} \overline{\bar{D}} \overline{\bar{P}}_{L}\right)^{-1} \\
\quad \times\left(\overline{\bar{P}}_{-L}^{\mathrm{T}} * \overline{\bar{M}}_{1}^{\mathrm{T} *} \bar{N}_{1}+\bar{P}_{L}^{\mathrm{T} *} \overline{\bar{D}}^{\mathrm{T} *} \overline{\bar{M}}_{2}^{\mathrm{T} *} \bar{N}_{2}\right) .
\end{aligned}
$$

The electrostatic free energy is

$$
\begin{aligned}
E=\frac{1}{16 L_{x}^{2} L_{y}^{2}} \overline{\hat{S}}_{-L}^{\mathrm{T} *} \cdot\left[\left(\overline{\bar{C}}_{1} \overline{\bar{P}}_{-L} \bar{v}_{\alpha \beta}^{*}\right) \star\left(\overline{\bar{C}}_{2} \overline{\bar{P}}_{-L} \bar{v}_{\alpha \beta}^{*}\right)\right] & \\
& +\frac{1}{16 L_{x}^{2} L_{y}^{2}} \overline{\bar{D}}_{q} \bar{S}_{L}^{T *} \cdot\left[\left(\overline{\bar{C}}_{1} \overline{\bar{P}}_{L} \bar{v}_{\alpha \beta}^{*} *\left(\overline{\bar{C}}_{2} \overline{\bar{P}}_{L} \bar{v}_{\alpha \beta}^{*}\right)\right] .\right.
\end{aligned}
$$

Equations [30] and [31] reveal that $\overline{\bar{D}}$ and $\overline{\bar{D}}_{q}$ are the only two terms in [31] which are functions of $d_{x}$ and $d_{y}$.

\section{RESULTS AND DISCUSSION}

In the numerical simulation we consider two identical, planar parallel surfaces. For illustration, only a two-dimensional problem is discussed. The period of the boundary condition is assumed to be 2 ; i.e., $2 L_{x}=$ 2 . Three types of boundary conditions are investigated: constant potential $\left(f_{1}=f_{2}=1, g_{1}=g_{2}=0, S_{-L}=1 / 2\right.$ and $\left.S_{L}=-1 / 2\right)$, constant charge density $\left(f_{1}=f_{2}=0, g_{1}=g_{2}=1, S_{-L}=-1 / 2\right.$ and $\left.S_{L}=1 / 2\right)$, and mixed boundary condition. The electrostatic repulsion energy is calculated by

$$
V_{r}(l)=E(l)-E(\infty) .
$$

The boundary conditions assumed are: (a) $\Psi=1,-1 \leqslant x \leqslant 1$; (b) $-\partial \Psi /$ $\partial \bar{n}=-1,-1 \leqslant x \leqslant 1$ (note that if this charge density is converted to potential, the result is the same as case (a)); (c) $\Psi=1$ for $-0.5 \leqslant x \leqslant$ 0.5 , and $-\partial \Psi / \partial \bar{n}$ is assigned the value of $-\partial \Psi / \partial \bar{n}$ in case (b) for $-1 \leqslant$ 
$x<-0.5$ and $0.5<x \leqslant 1$. Figure 1 shows the boundary condition assumed for case (c). The variations in the electrostatic repulsion energy $V_{\mathrm{r}}$ as a function of half the separation distance between two surfaces $L$ for cases (a) - (c) are pictured in Fig. 2. This figure reveals that $V_{\mathrm{r}}$ increases with the decrease in $L$ for three types of boundary condition. For a fixed $L, V_{\mathrm{r}}$ follows the order (constant charge density) $>$ ( mixed condition) $>$ (constant potential). If $L$ is greater than the Debye length, $V_{\mathrm{r}}$ is about the same, regardless of the types of boundary conditions assumed. Note that for a fixed $L, V_{\mathrm{r}}$ is minimized at $d x=1$ for the case of mixed condition. In other words, a shift in one of the two surfaces with a half period leads to a minimum in $V_{\mathrm{r}}$ for this example. This is consistent with the results of Kuin (7) and Vreeker et al. (8), in which only the leading terms of the righthand side of [12] are considered. Note that the minimum of $V_{\mathrm{r}}$ does not necessarily occur at a shift of half the period, in general. It depends largely on the type of boundary condition specified and the number of terms adopted in [12]. We show that two approaching surfaces may adjust their conditions and relative position to minimize the interaction energy. Thus, when two charge-regulated particles, each having a uniform surface condition, come close to each other, their surface conditions can be of a complicated nature (7-9). It is possible that part of a surface remains at constant potential and the rest at constant charge density. This leads to a mixed boundary value problem. Another possible case is the interaction of two porous particles. Here, the solid-liquid interface may remain at constant charge while the interparticle liquid-pore-liquid interface is at approximately constantpotential.

The criterion for the estimation of the unknown coefficients in [12] is based on the frequency domain expanded by $(p, q)$. An equivalent criterion in the $(x, y)$ domain can be found by applying Plancherel's identity (3). The latter, however, is not readily applicable for the estimation of the effect of position shift on the electrostatic interaction. In this case a nontrivial numerical scheme is required, in general.

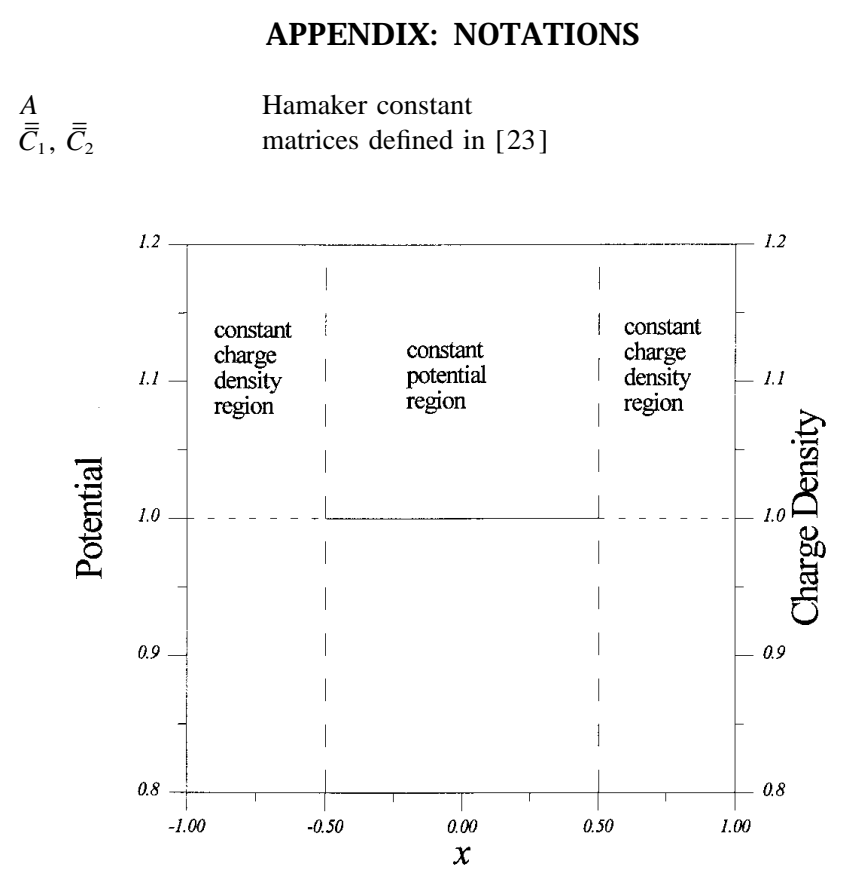

FIG. 1. The boundary condition assumed in the illustrating example. The potential is fixed in the region $-0.5 \leqslant x \leqslant 0.5$, and the surface charge density is fixed in the region $-1 \leqslant x<-0.5$ and $0.5<x \leqslant 1$. The solid line denotes the distribution of potential described by $\Psi=1$, and the dashed line represents the distribution of charge density, described by $-\partial \Psi / \partial \bar{n}=1$.

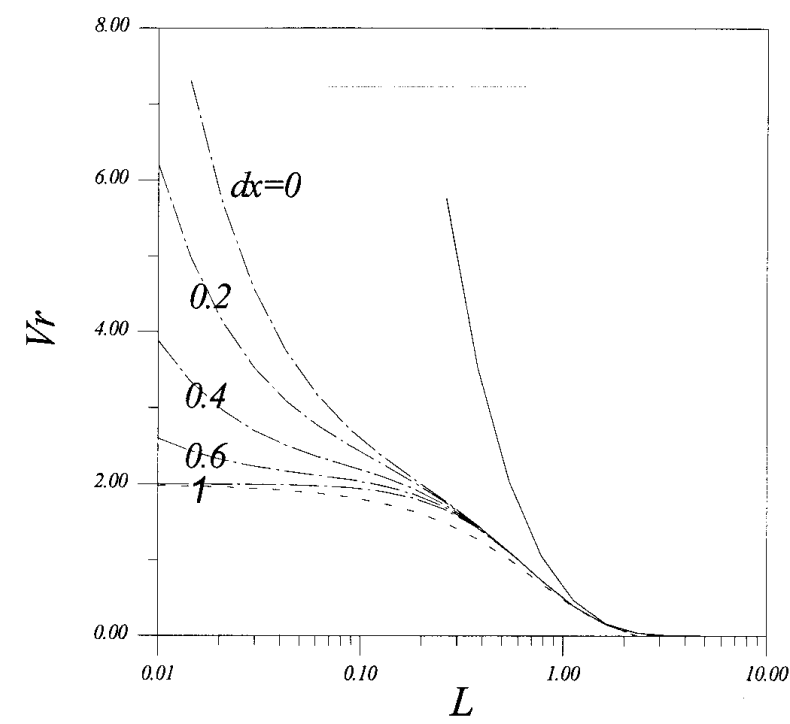

FIG. 2. Variation in the electrostatic repulsion energy as a function of half the separation distance between two surfaces for three types of boundary conditions at various shift distances. (- - ) constant potential; $(-)$ constant charge density; $(-\cdot-)$ mixed conditions. The boundary condition for the last case is shown in Fig. 1.

$d_{x}, d_{y}$
$D_{p, q}$
$\overline{\bar{D}}, \overline{\bar{D}}_{p}$
$e$
$E$
$I_{p q}$
$k$
$L$

$L_{x}, L_{y}$

$L_{x}, L_{y}$
$\overline{\bar{M}}_{1}, \overline{\bar{M}}_{1}, \bar{v}_{L}, \bar{v}_{L, k}$,
$\quad \bar{N}_{1}, \hat{h}_{1, k}, \bar{N}_{2}, \hat{h}_{2, k}$
$n_{0}$
$\overline{\bar{P}}_{-L, p q}, \overline{\bar{P}}_{L, p q}$

$T$

$V_{\mathrm{r}}$

$x, y, z$

$z_{i}$

$\tilde{x}, \tilde{y}, \tilde{z}$

\section{Greek Letters}

$\alpha_{p q}, \beta_{p q}$
$\epsilon_{0}$
$\epsilon_{\mathrm{r}}$
$\Phi$
$\kappa$
$\psi(p, x), \psi(p, y)$
$\Psi$

shifted units in the $x$ and $y$ directions, respectively shift factor defined below [28]

matrices defined below [29]

elementary charge

dimensionless energy defined in [21]

coefficient defined in [3]

Boltzmann constant

half the dimensionless separation distance between two surfaces

half periods in the $x$ and $y$ directions, respectively

defined below [14]

bulk number concentration of ions

transform matrices defined in [7] and [11], respectively

temperature

dimensionless electrical repulsion energy defined in [32]

dimensionless position variable

the valence of ion species $i$

position variable

coefficients defined in [3]

permittivity of the vacuum

relative permittivity of solution

electrical potential

reciprocal Debye length

orthogonal functions defined in [3]

dimensionless potential 


\section{REFERENCES}

1. Ninham, B. W., and Parsegian, V. A., J. Theor. Biol. 31, 405 (1971).

2. Carnie, S. T., Chan, D. Y. C., and Stankovich, J., J. Colloid Interface Sci. 165, 116 (1994).

3. Kwakernaak, H., and Sivan, R., ' 'Modern Signals and Systems.' Prentice Hall, Englewood Cliffs, NJ, 1990.

4. Hunter, R. J., 'Foundations of Colloid Science,' Vol. I. Oxford Univ. Press, London, 1989.

5. Lyklema, J., "Fundamentals of Interface and Colloid Science.' Academic Press, London, 1991.

6. Ohshima, H., Adv. Colloid Interface Sci. 53, 77 (1994).

7. Kuin, A. J., J. Chem. Soc. Faraday Discuss. 90, 235 (1990).

8. Vreeker, R., Kuin, A. J., Den Boer, D. C., Hoekstra, L. L., and Agterof, W. G. M., J. Colloid Interface Sci. 145, 138 (1992).
9. Miklavic, S. J., Chan, D. Y. C., White, L. R., and Healy, T. W., J. Phys. Chem. 98, 9022 (1994).

Ming-TSAN TSENG

JYH-PING HsU ${ }^{1}$

Department of Chemical Engineering

National Taiwan University

Taipei, Taiwan 10617

Republic of China

Received October 13, 1995

${ }^{1}$ To whom correspondence should be addressed. 